OPEN ACCESS

Edited by:

Ruoyu Wang,

University of Edinburgh,

United Kingdom

Reviewed by:

Haoran Yang,

East China Normal University, China

Bin Jiang,

The University of Hong Kong,

Hong Kong

*Correspondence:

Ran Barzilay

barzilayr@chop.edu;

ran.barzilay@pennmedicine.upenn.edu

Specialty section

This article was submitted to

Health and Cities,

a section of the journal

Frontiers in Sustainable Cities

Received: 26 March 2021

Accepted: 31 May 2021

Published: 26 July 2021

Citation:

Wortzel JD, Wiebe DJ

DiDomenico GE, Visoki E, South $E$

Tam V, Greenberg DM, Brown LA, Gur RC, Gur RE and Barzilay R (2021)

Association Between Urban

Greenspace and Mental Wellbeing

During the COVID-19 Pandemic in a

U.S. Cohort.

Front. Sustain. Cities 3:686159.

doi: $10.3389 /$ frsc. 2021.686159

\section{Association Between Urban Greenspace and Mental Wellbeing During the COVID-19 Pandemic in a U.S. Cohort}

\author{
Jeremy D. Wortzel ${ }^{1}$, Douglas J. Wiebe ${ }^{2}$, Grace E. DiDomenico ${ }^{1,3}$, Elina Visoki ${ }^{1,3}$, \\ Eugena South ${ }^{4,5}$, Vicky Tam ${ }^{2,6}$, David M. Greenberg ${ }^{7}$, Lily A. Brown ${ }^{8}$, Ruben C. Gur ${ }^{3,8}$, \\ Raquel E. Gur ${ }^{3,8}$ and Ran Barzilay ${ }^{3,8,9 *}$

\begin{abstract}
${ }^{1}$ Perelman School of Medicine, University of Pennsylvania, Philadelphia, PA, United States, ${ }^{2}$ Department of Biostatistics, Epidemiology and Informatics, Perelman School of Medicine, University of Pennsylvania, Philadelphia, PA, United States, ${ }^{3}$ Lifespan Brain Institute, Children's Hospital of Philadelphia \& Penn Medicine, Philadelphia, PA, United States, ${ }^{4}$ Department of Emergency Medicine, Perelman School of Medicine, University of Pennsylvania, Philadelphia, PA, United States, ${ }^{5}$ Urban Health Lab, Perelman School of Medicine, University of Pennsylvania, Philadelphia, PA, United States, ${ }^{6}$ Healthcare Analytics Unit, Department of Biomedical and Health Informatics, The Children's Hospital of Philadelphia, Philadelphia, PA, United States, ${ }^{7}$ Interdisciplinary Department of Social Sciences, Bar-llan University, Ramat Gan, Israel, ${ }^{8}$ Department of Psychiatry, Perelman School of Medicine, University of Pennsylvania, Philadelphia, PA, United States, ${ }^{9}$ Department of Child and Adolescent Psychiatry, Children's Hospital of Philadelphia, Philadelphia, PA, United States
\end{abstract}

The Coronavirus Disease 2019 (COVID-19) pandemic brought unprecedented socially isolating measures to mitigate the spread of disease, heightening the importance of public outdoor urban greenspace. Here, we investigated the association between tree-rich greenspace and mental health in a large opportunity sample surveyed using a crowdsourcing research website (www.covid19resilience.org) between April 6th and October 12th, 2020 during the pandemic in the United States. Participants living in the U.S. ( $N=2,089,83.1 \%$ females, mean age 42 years, age range 18-90 years) were mapped to 1,080 unique ZIP Codes and mean tree canopy density was calculated with a $250 \mathrm{~m}$ buffer around each ZIP Code grouped by quartile as a proxy for nearby greenspace. Four mental health parameters were assessed: (1) COVID-19-related worries; (2) anxiety symptoms; (3) depression symptoms; and (4) a standardized and weighted composite mental health score of all three parameters. Multivariate regression analyses with multilevel models were used to study the association between nearby greenspace and the four mental health outcomes, controlling for participant demographics and ZIP Code urbanicity. In the entire cohort, increased nearby greenspace showed a significant protective effect for depression (Coef. $=-0.27 ; p=0.0499$ ), and composite mental health scores (Coef. $=-0.19$; $p=0.038$ ) when comparing ZIP Codes with the most greenspace to ZIP Codes with the least, with no observed effects on COVID-19 related worries or anxiety individually. Stratifying by age suggested protective trends of greenspace on mental health in older subsets of the population (top age quartile, over 51 years old) experiencing less depression (Coef. $=-0.45 ; p=0.048$ ) and lower composite mental health scores (Coef. $=-0.34 ;-=0.032$ ) as a function of nearby greenspace. Additionally, younger 
subsets of the population (second youngest age quartile, 31-38 years) experienced lower COVID-19 related worries (Coef. $=-1.34 ; p=0.022$ ) as a function of greenspace. These findings may indicate that tree-rich greenspace plays a protective role on mental health during the COVID-19 pandemic, specifically in certain age groups, supporting the use of greenspace-related strategies to help mitigate mental health burden during this challenging and isolating time.

Keywords: urban greenspace, COVID-19, nature-based solutions, personalized ecology, mental health

\section{INTRODUCTION}

Throughout the Coronavirus Disease 2019 (COVID-19) pandemic, actions were taken by countries around the world to limit the spread of the virus. These actions included requiring lockdown measures, social distancing precautions, and virtual school and work alternatives to limit human interactions. In the United States, the first mitigation strategies began in March 2020 (Moreland et al., 2020). While beneficial for disease prevention, these mitigation efforts have been associated with negative mental health outcomes as symptoms of depression and anxiety worsened in the general population over the course of this pandemic (Marchini et al., 2020; Panchal et al., 2020; Rossi et al., 2020; Vindegaard and Benros, 2020).

At the same time, individuals frequented greenspaces more readily. For example, a Norwegian study estimated that outdoor recreational activity increased by $291 \%$ during lockdown relative to the 3-year prior average for the same days (Venter et al., 2020). In some areas, increased greenspace utilization was used strategically in direct response to increased disease prevalence. For example, in several East Asian cities, greenspace utilization increased by $5.3 \%$ for every 100 additional new COVID-19 cases reported (Lu et al., 2021b). The cause of increased greenspace utilization is multifactorial. Not only did outdoor space provide an escape from home isolation (Naomi, 2020), but also outside spaces emerged as a relatively safe method to socialize with others, an activity which became restricted anywhere aside from the outdoors (Killgore et al., 2020; Marchini et al., 2020).

Additionally, spending more time in greenspace during the pandemic may have influenced emotional wellbeing, as a growing body of pre-pandemic evidence suggests that living near and spending time in greenspace is associated with better mental health (Twohig-Bennett and Jones, 2018). Increased usable urban greenspace was associated with decreased anxiety and mood disorders (Nutsford et al., 2013) as well as lower risks of developing psychiatric disorders in adulthood (Engemann et al., 2019). Observational studies established similarly strong associations between greenspace and mental health in urban environments in the United States and abroad (Beyer et al., 2014; Ruijsbroek et al., 2017). A recent randomized control trial examining the impact of interventions to green vacant urban land found that self-reported feelings of depression and worthlessness were significantly decreased in those living next to newly greened lots, compared to those living around lots that remained blighted (South et al., 2018). However, all greenspace does not impact mental health equally. Tree canopy, over other forms of urban greenery such as open grass spaces, was most strongly associated with better mental health outcomes (Astell-Burt et al., 2014), as well as with perceptions of overall greenspace (Astell-Burt et al., 2014; Astell-Burt and Feng, 2019; Mazumdar et al., 2020).

While the association between greenspace and improved mental health is well-established, and greenspace utilization during COVID-19 increased, only a few studies have investigated the relationship of greenspace and mental health during the COVID-19 pandemic. Studies thus far found evidence to support that individuals with accessible outdoor spaces or with natural elements in window views while in quarantine experienced decreased symptoms of depression and anxiety with improved measures of mental health (Pouso et al., 2020; Slater et al., 2020; Soga et al., 2020). The current study aims to further this work to determine the association between nearby tree-rich greenspace and mental health during the COVID-19 pandemic in a U.S. cohort. We hypothesized that having trees located nearby an individual's home neighborhood positively influenced their emotional wellbeing during this pandemic.

\section{MATERIALS AND METHODS}

\section{General Approach}

This study explores the association between tree-rich greenspace in ZIP Codes and four measures of mental health during the COVID-19 pandemic: COVID-19-related worries, anxiety, depression, and a weighted composite score generated from all three measures. We analyzed data from 2,089 individuals surveyed through a research crowdsourcing website (www.covid19resilience.org) between April 6th and October 12th, 2020. Participants were living in 1,080 unique ZIP Codes across the United States. Building on previous findings from this data source, we sought to disentangle the specific association between greenspace defined by tree canopy density and mental health during the pandemic from other factors related to mental health including age, race, sex, and education status (Barzilay et al., 2020); as well as stressors uniquely impacting mental health during this pandemic such as living alone (Gao and Sai, 2020), and recent job loss (Ziersch et al., 2014), Additionally, because COVID-19 has differentially impacted mental health according to age (Banerjee, 2020; Barzilay et al., 2020) we explored greenspace impacts at different ages across the lifespan.

\section{Participants and Procedures}

Participants for this study were sampled from an online survey (www.covid19resilience.org) launched in April 2020 
to study resilience and mental health during the COVID-19 pandemic (Barzilay et al., 2020). In addition to providing data on demographics, COVID-19 exposures and mental health, participants living in the United States also indicated their ZIP Code. The survey collected demographic information including mental health history and factors influencing mental health during the pandemic (such as living alone and recent job loss), as well as three measures of current mental health. First, participants were asked to complete an interactive assessment of COVID-19-related stress (worries) regarding: (1) getting (contracting), (2) dying from, (3) currently having, (4) family member getting, (5) unknowingly infecting others with, and (6) experiencing significant financial burden following COVID19. A 5-item rating scale $(0-$ not at all; $1-$ a little; $2-a$ moderate amount; 3-a lot; 4-a great deal) was provided to allow participants to indicate how much each parameter concerned them. Next, participants were offered to take a second survey on their anxiety symptoms [generalized anxiety disorder 7 questionnaire (GAD7)] (Spitzer et al., 2006) and depression symptoms [patient health questionnaire 2 (PHQ2)] (Arroll et al., 2010). Participants received feedback on several components of their responses with personalized recommendations regarding stress management. The feedback was also meant to incentivize participants to complete the survey accurately. The study was advertised through: (1) the researchers' social networks, including emails to colleagues; (2) social media; (3) the University of Pennsylvania and Children's Hospital of Philadelphia internal notifications; and (4) organizational mailing lists. The results presented here are based on data collected from participants living in the United States. Participation required responders to provide online consent. The study was approved by the Institutional Review Board of the University of Pennsylvania. All data was processed and cleaned using RStudio version 1.1.453 (R Studio Team, 2020).

\section{Measures of Mental Health}

We used principal-components factor analysis (Joliffe, 1992) to examine whether responses to the three mental health measures were correlated and thus appropriate to consider in combination as a single mental health construct. The motivation to do so stems from the work of other researchers, who have described how external forms of stress, such as the COVID-19 pandemic can potentiate underlying anxiety and depression (Gobbi et al., 2020). Doing so revealed one distinguishable factor with an eigenvalue of 1.95 formed by COVID-19-Related Worries, GAD7, and PHQ-2. The factors loadings for the three mental health measures were all over $0.6(0.67,0.89,0.83$, respectively). Finding this evidence of a single construct, we generated a new composite variable called Composite Mental Health Score, derived as the weighted sum of responses to the three measures in standardized form (mean $=0, S D=1)$.

\section{Measure of Greenspace}

Greenspace was measured through mean tree canopy density with a $250 \mathrm{~m}$ buffer around each ZIP Code divided into quartiles. This proxy was chosen because (1) tree canopy, over other forms of urban greenery, has been most strongly associated with improved mental health outcomes (Astell-Burt et al., 2014); (2) other forms of urban greenery, such as grass spaces, have been associated with worse mental health outcomes (AstellBurt and Feng, 2019); and (3) tree canopy density was shown to be the most acuate proxy for total perceived greenspace when compared to other common spatial measures including percent parks and Normalized Differential Vegetative Index (NDVI) (Mazumdar et al., 2020). Therefore, throughout this study the term greenspace will be used to refer to tree-rich greenspaces exclusively created by tree canopy as seen from areal imaging. Additionally, buffers surrounding ZIP Codes have been utilized in other studies to depict the viewable and walkable neighborhood especially for individuals living on the boundaries of a particular ZIP Code (Cox et al., 2017; Soga et al., 2020). We selected a $250 \mathrm{~m}$ buffer as this is the distance considered to influence the experiences of a person in their daily life and has been shown to be the threshold distance for appreciable physical and mental benefits of greenspace around a ZIP Code (Browning and Lee, 2017; Cox et al., 2017). Additionally, in preliminary analysis, several buffer sizes were considered (e.g., $100,500,1,000 \mathrm{~m}$ ) which confirmed that the buffer size did not essentially affect the results of the study as described in Table A1.

Mean tree canopy density was calculated using the United States Department of Agriculture (USDA) Forest Service 2016 Tree Canopy Cover for the continuous United Stated (Bender et al., 2019). Tree Canopy included tree leaves, needles, and branches that provide tree coverage of the ground, viewed from an aerial perspective. This USDA product provided a raster image of the continuous United Stated broken down into individual pixels representing $30 \times 30 \mathrm{~m}$ squares of land. Each pixel was identified with a tree canopy cover value, ranging from 0 to 100 , reflecting the tree canopy density of that area. Using ArcMap version 10.8 (ESRI, 2011), all pixel values within a ZIP Code including a $250 \mathrm{~m}$ buffer were averaged creating a mean tree canopy density value used in this study as a proxy for greenspace. ZIP Codes were then stratified by quartiles from low to high mean tree canopy density: Q1 $(<6.90 \%)$, Q2 (6.91-18.61\%), Q3 (18.62-32.12\%), Q4 (>32.13\%). These greenspace quartiles acted as the primary independent variable throughout the analyses.

\section{Statistical Analysis Main Analysis}

Descriptive statistics were used to summarize the data. Variance inflation factors (VIF) were calculated to explore the potential for multicollinearity, and found to be $<3$, supporting the assumption that the variables were statistically independent and suitable to be modeled simultaneously as covariates. Multivariable regression was used to evaluate the association between greenspace in each ZIP Code and mental health. Due to the hierarchical nature of the data, with geographic nesting of survey participants, multilevel models with random intercepts for ZIP Codes was used to avoid the potential for bias (Bryk and Raudenbush, 1992). Doing so was supported by the intra-class correlation coefficients of the models (i.e., 0.016-0.032), showing that mental health measures in the same ZIP Codes were somewhat correlated. 
The regression modeling included all participants and involved four multilevel models-one for each of the four mental health outcomes: (1) COVID-19-related worries (continuous scores ranging from 0 to 24 derived from an aggregate of all six worry questions rated on a 5-point scale from 0 to 4), (2) GAD-7 scores (continuous, ranging from 0 to 21), (3) PHQ-2 scores (continuous, ranging from 0 to 6), and (4) a Composite Mental Health Score (continuous weighted score comprised of all three mental health measures including COVID19 related worries, GAD-7 and PHQ-2, ranging from -1.78 to 3.35). Greenspace divided by quartile was the independent variable of interest in each model, with the quartile with the least greenspace (Q1) treated as the reference group. Covariates in each model were participant gender, age, race, education status, prior mental health diagnosis, recent job loss, living alone, and urbanicity of the participant's ZIP Code of residence. All covariates were analyzed as categorical variables accounting for non-linear associations with each outcome. Prior mental health diagnosis was determined for participants who endorsed being diagnosed with a mental health condition by a professional prior to the pandemic. Urbanicity was defined by the 2013 Rural Urban Continuum Codes (RUCC) which form classification schemes for U.S. counties utilizing population size and adjacency to metro areas to determine urbanicity (scale from 1 to 8 ; most urban to most rural) (Rural-Urban Continuum Codes, 2013). Annual household income was included in all initial models, but excluded from final analyses for parsimony after finding no instances of being statistically associated with mental health. All regression analyses were conducted in Stata/IC version 16.1 (StataCorp, 2019).

\section{Secondary Analysis}

Given that the relation between mental health and greenspace may vary as a function of age, we stratified the cohort by age quartiles and ran the multilevel models as described above within each age-group quartile. Age quartiles, rather than small groupings, were used in an attempt to have sample sizes adequate and precision in the effect estimates. All regression analyses were conducted in Stata/IC version 16.1 (StataCorp, 2019).

\section{RESULTS}

\section{Spatial Distribution of Study Participants}

The survey data were comprised of 2,089 participants (surveyed April 6th through October 12th, 2020) who provided ZIP Code of residence and completed the COVID-19-related worries screening questions. The majority of participants were female $(n=1,736,83.1 \%)$ and identified as White $(n=1,802,86.3 \%)$. Age ranged considerably, from 18 to 90 years (mean age $=42$ years, standard deviation $=13.4$ ). Full demographics are shown in Table 1.

Figure 1A shows a visualization of all U.S ZIP Codes and ZIP Codes of all study participants, with a close-up of the number of surveyed participants in the greater Philadelphia area in Figure 1B, to show an example of the geographic specificity. The majority of participants were located in urban centers or immediate metro-adjacent ZIP Codes (RUCC 1 and 2; $n=969$,
TABLE 1 | Cohort demographics ${ }^{\mathrm{a}}(N=2089)$.

\begin{tabular}{lcc}
\hline & $n$ & $\%$ \\
\hline Age bins, years $^{\text {b }}$ & & \\
18-30 & 468 & 22.4 \\
$31-38$ & 422 & 20.2 \\
$39-51$ & 506 & 24.2 \\
$51-90$ & 478 & 22.9 \\
Other demographics & & \\
Gender, female & 1,736 & 83.1 \\
Gender, male & 348 & 16.7 \\
Race, white & 1,797 & 86.7 \\
Race, non-white & 276 & 13.3 \\
Income (annual per household) & & \\
Under $\$ 50,000$ & & 15.1 \\
\$50,000 to $\$ 74,999$ & 315 & 14.6 \\
\$75,000 to $\$ 149,999$ & 304 & 32.1 \\
\$150,000 and above & 670 & 31.2 \\
Mental health risk factors & 652 & \\
Prior mental health diagnosis & & 35.5 \\
Recent job loss & 1,168 & 0.04 \\
Lives alone & 83 & 21.2 \\
\hline
\end{tabular}

${ }^{a}$ Missing demographic data for participants answering "I don't know/l'd rather not say" was lower than $1.8 \%$ for all variables except age and income.

${ }^{b}$ Missing data for age $=10.3 \%$.

${ }^{c}$ Missing data for household income $=7.1 \%$.

91.8\%) as shown in Figure 1D. The distribution of greenspace for study participants across the U.S. is summarized in Figure 1C with the proportion of greenspace for each study participant represented in Figure 1E.

\section{Factors Associated With Mental Health}

When assessing the entire cohort, greenspace showed a significantly protective effect on clinical depression scores (PHQ2 Coef. $-0.27 ; p=0.0499)$ and Composite Mental Health Scores (Coef. $=-0.19 ; p=0.038$ ) when comparing ZIP Codes with the most greenspace (Q4) to the ZIP Codes with the least greenspace (Q1). Greenspace was not associated with COVID-19 related worries $(p$-value $>0.097)$ or with anxiety $(P$-values $>0.064)$ when assessing the entire cohort. Details of the results of how greenspace related to the mental health variables in the entire cohort are reported in Table 2. Results on how the covariates including race, gender, education status, prior mental health diagnosis, living alone, and urbanicity related to the mental health outcomes are reported in Table A2, but are not discussed here given the results are consistent with findings that were presented previously in which their relation to the pandemic was the focus (Barzilay et al., 2020).

\section{Stratified Exploratory Analyses}

Table 3 reports results of the models stratified by age groups to explore the association between greenspace and mental health across the lifespan. In the oldest age group (individuals between 52 and 90 years old), greenspace was associated with lower 

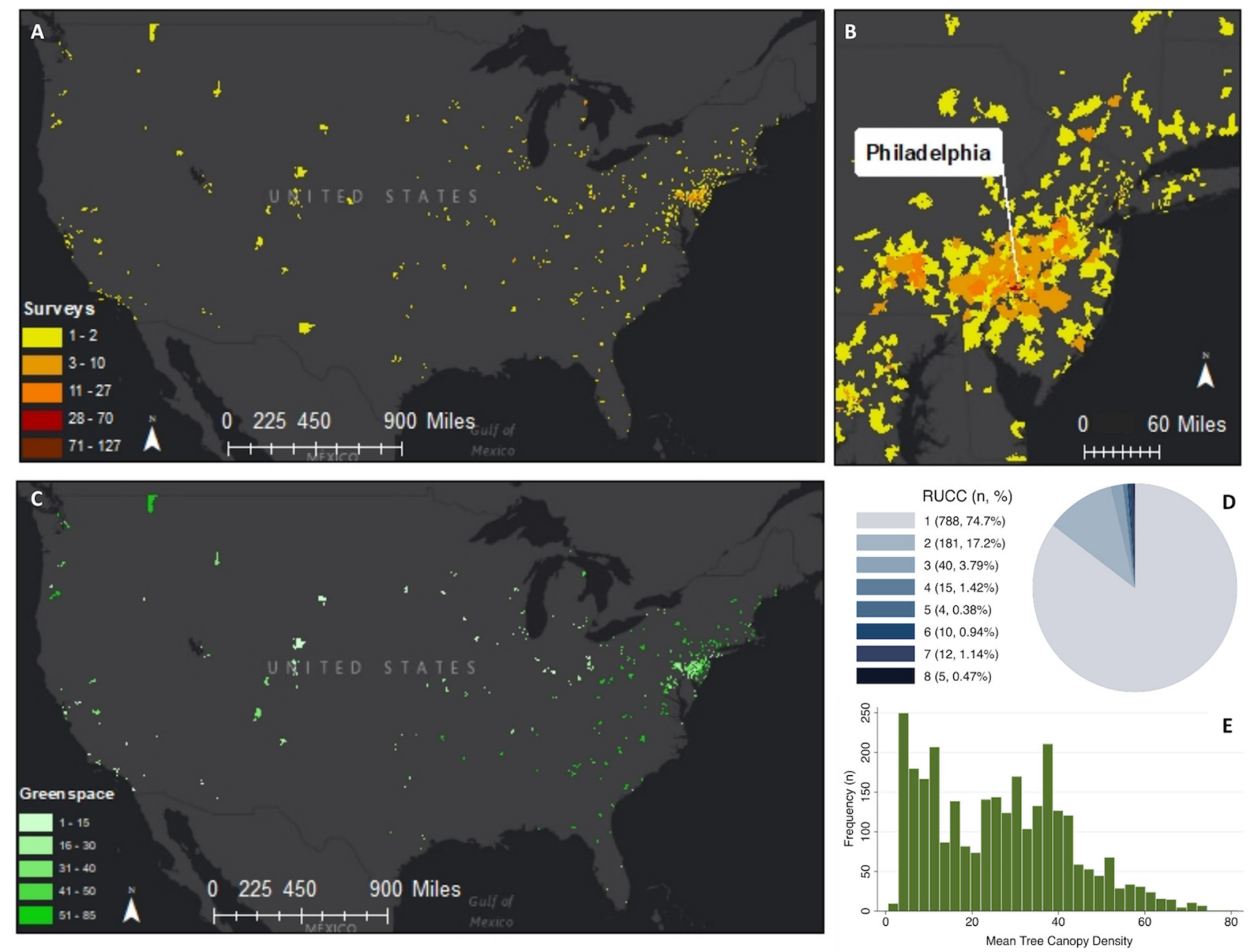

FIGURE 1 | Geospatial analysis of survey data and greenspace showing: (A) the distribution of the surveyed population across ZIP Codes throughout the United States; (B) a close-up of the surveyed population in the greater Philadelphia area; (C) estimated greenspace in all surveyed ZIP Codes. Note ZIP Codes with 0\% green space do not appear on the map; (D) The distribution of urbanicity measured by Rural-Urban Continuum Codes (RUCC) for all surveyed ZIP Codes ranked 1 (most urban) to 8 (most rural); (E) The distribution of greenspace in the surveyed population. Base-map Credits: Esri.

depression scores (PHQ-2, Coef. $=-0.45 ; p=0.048)$ and lower Composite Mental Health Scores (Coef. $=-0.34 ; p=0.032$ ) when comparing ZIP Codes with the most greenspace to the ZIP Codes with the least greenspace (Q4 vs. Q1). Additionally, higher greenspace was associated with lower COVID-related worries (Coef. $=-1.34 ; p=0.022$ ) in individuals in the second youngest age group (ages 31-38 yo) when comparing individuals who lived in ZIP Codes with the second largest amount of greenspace to those with the least (Q3 vs. Q1). Clinical measures of anxiety (GAD-7) were not statistically associated with greenspace $(P$ values $>0.068$ ).

\section{DISCUSSION}

In this cross-sectional analysis of an opportunity sample conducted in the U.S. during the first six-months of the COVID-19 pandemic, we found that greenspace showed significant protective effects on both depression and composite mental health scores of the entire cohort. Exploratory analysis showed that in older populations, proximity to tree-rich greenspace was associated with decreased depression and Composite Mental Health Scores and in younger populations greenspace was associated with lower COVID-19 related worries. The fast spread of COVID-19, and the unique socially isolating precautions that were implemented, creates an opportunity to evaluate the impact that greenspace has on mental health. Here, we leverage a resource that collected data on COVID-19 stress and mental health and enables ZIP Code-based estimation of greenspace, allowing us to explore the impact of greenspace on several measures of mental health during this pandemic. Our findings support the prior literature on the benefits of greenspace on mental health, and expand on the scarce data on this topic during the pandemic. 
TABLE 2 | Greenspace and COVID-19-related worries, anxiety, depression, and composite mental health scores among all surveyed participants, multivariate regression analysis with multilevel models.

\begin{tabular}{|c|c|c|c|c|c|}
\hline \multirow[t]{2}{*}{ Study population } & \multirow[t]{2}{*}{ Greenspace quartiles } & $\begin{array}{l}\text { COVID-19 related worries } \\
\qquad\left(n=1,874^{\mathrm{a}}\right)\end{array}$ & $\begin{array}{c}\text { GAD-7 } \\
\left(n=1,129^{a}\right)\end{array}$ & $\begin{array}{c}\text { PHQ-2 } \\
\left(n=1,129^{a}\right)\end{array}$ & $\begin{array}{l}\text { Composite mental health score } \\
\qquad\left(n=1,129^{a}\right)\end{array}$ \\
\hline & & Coef. $(95 \%$ Cl) & Coef. $(95 \%$ Cl) & Coef. $(95 \% \mathrm{Cl})$ & Coef. (95\% Cl) \\
\hline \multirow[t]{6}{*}{ Entire cohort } & Q2 vs. Q1 & -0.18 & -0.41 & -0.077 & -0.077 \\
\hline & & $(-0.79,0.44)$ & $(-1.31,0.49)$ & $(-0.34,0.19)$ & $(-0.25,0.095)$ \\
\hline & Q3 vs. Q1 & $-0.53^{\dagger}$ & $-0.79^{\dagger}$ & -0.033 & -0.13 \\
\hline & & $(-1.16,0.095)$ & $(-1.69,0.12)$ & $(-0.29,0.23)$ & $(-0.31,0.038)$ \\
\hline & Q4 vs. Q1 & -0.33 & $-0.85^{\dagger}$ & $-0.27^{\star}$ & $-0.19^{*}$ \\
\hline & & $(-0.96,0.29)$ & $(-1.75,0.05)$ & $(-0.53,-0.0001)$ & $(-0.36,-0.017)$ \\
\hline
\end{tabular}

GAD-7, Generalized Anxiety Disorder Questionnaire 7-item; PHQ2, Patient Health Questionnaire- 2 item; Cl, confidence interval.

${ }^{a}$ The number of participants in each group is lower in multilevel model relative to Table $\mathbf{1}$ as only those participants who responded to all variables were included in the analysis.

${ }^{\dagger} P<0.1,{ }^{*} p<0.05$.

A major finding in this study is that increased greenspace was associated with lower depression symptoms and lower composite mental health scores. These findings are in line with well-established, pre-pandemic protective associations between greenspace and clinical measures of depression (Nutsford et al., 2013; Ruijsbroek et al., 2017; Engemann et al., 2019; Hedblom et al., 2019) as well as with findings of recent studies on this topic conducted during COVID-19 (Pouso et al., 2020; Soga et al., 2020). This study supports that in times of isolation and quarantine, greenspaces, specifically those with tree canopy, become important factors at maintaining mental health. The pandemic may have increased the importance of greenspaces, as they not only provide tranquil respites from urban streetscapes, but also uniquely serve as social gathering locations for those observing social distancing precautions. Therefore, we echo the growing recommendations for making parks and greenspace more accessible in order to preserve mental health, now and in future pandemics (Slater et al., 2020). While this association was not statistically seen with anxiety or COVID-19-related worries within the entire cohort of our study, some effect estimates were trending toward significance and more research is required to understand the nuance of these trends.

Another finding of this study is that age may play a role in how individuals benefit from greenspace. Both sides of the age spectrum seem to show some benefit, while similar trends in middle-aged individuals were not observed. There are several possible explanations for these findings. First, prior studies have shown some age discrepancy regarding how greenspace is appreciated over a life course, with increased benefits of greenspace later in life (Astell-Burt et al., 2014). This finding may be attributed to several factors including generational differences in nature appreciation or the quality of greenspace itself. Prior studies showed that while greenspace in and of itself has been beneficial to mental health (Hedblom et al., 2019), the quality of greenspace is also a substantial factor in the perceived enjoyment (Francis et al., 2012). Older individuals may have more resources like time, transportation, and better living arrangements relative to younger individuals and therefore may have access to private or better-quality greenspaces. Perhaps the quality of greenspace including the landscaping maintenance, aesthetic values, biodiversity levels, and tranquility of green spaces are all important to consider in future studies and in future urban greening interventions.

We also found that the mental health of the second youngest age group (ages 31-38 yo) was more impacted by greenspace relative to the mental health of the youngest age group (1830 yo) and that of the second oldest age group (39-51 yo). There are several COVID-19 specific considerations that may explain this unique trend. For example, prior studies noted that having school-aged children during this pandemic has negatively impacted measures of mental health in the parents because school closures led to more stress at home and higher pressures while working remotely (Hiraoka and Tomoda, 2020). This may be especially true for the parent who takes on primary caregiver responsibilities. Therefore, working adults with schoolaged children, as opposed to younger adults without children or older non-working retired adults, may have had added stressors and reported more worries during this time. These stressors may also contribute to the amount of time a person has to engage with the outdoors and with greenspaces in their area thereby impacting the influence of greenspaces on their mental wellbeing.

Additionally, even though COVID-19 was disproportionately impacting the health of older individuals who therefore may have had a higher perceived knowledge of their vulnerability and overall threat of COVID-19 (Banerjee, 2020), adolescents and middle-aged adults had worse mental health outcomes during the pandemic (Barzilay et al., 2020; Ueda et al., 2020). Though little research has been done to examine this discrepancy, financial, and job instability (Witteveen and Velthorst, 2020) in the working-age population and decreased social support structures for younger individuals (Cowie and Myers, 2021) are possible explanations. Adolescents have been singled out as a group particularly impacted by isolation due to the inherent lack of independence during quarantine. However, young adults who have already established their independence and social supports, yet do not have the responsibilities of school-aged children, may potentially be less effected and contribute to our findings (Ravens-Sieberer et al., 2021). Although we controlled for some 


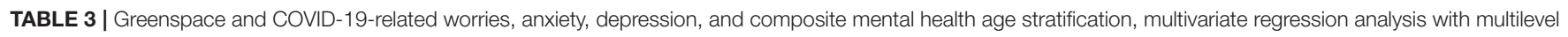
models.

\begin{tabular}{|c|c|c|c|c|c|}
\hline \multirow[t]{2}{*}{$\begin{array}{l}\text { Study population } \\
\text { (Age quartiles) }\end{array}$} & \multirow[t]{2}{*}{$\begin{array}{l}\text { Greenspace } \\
\text { quartiles }\end{array}$} & \multirow{2}{*}{$\begin{array}{c}\text { COVID-19 } \\
\text { related worries } \\
\text { Coef. }(95 \% \mathrm{CI})\end{array}$} & GAD-7 & PHQ-2 & \multirow{2}{*}{$\begin{array}{c}\begin{array}{c}\text { Composite mental } \\
\text { health score }\end{array} \\
\text { Coef. }(95 \% \mathrm{Cl})\end{array}$} \\
\hline & & & Coef. $(95 \%$ Cl) & Coef. (95\% CI) & \\
\hline \multirow{3}{*}{$\begin{array}{l}\text { Age quartile } 1 \\
(18-30 \text { yo) } \\
N=468^{a}\end{array}$} & Q2 vs. Q1 & $\begin{array}{c}0.33 \\
(-0.64,1.31)\end{array}$ & $\begin{array}{c}-0.0001 \\
(-1.75,1.74)\end{array}$ & $\begin{array}{c}0.24 \\
(-0.27,0.75)\end{array}$ & $\begin{array}{c}0.094 \\
(-0.21,0.39)\end{array}$ \\
\hline & Q3 vs. Q1 & $\begin{array}{c}0.41 \\
(-0.70,1.53)\end{array}$ & $\begin{array}{c}-0.59 \\
(-2.51,1.32)\end{array}$ & $\begin{array}{c}0.23 \\
(-0.35,-0.80)\end{array}$ & $\begin{array}{c}0.018 \\
(-0.33,0.37)\end{array}$ \\
\hline & Q4 vs. Q1 & $\begin{array}{c}0.89 \\
(-0.28,2.06)\end{array}$ & $\begin{array}{c}0.066 \\
(-1.96,2.09)\end{array}$ & $\begin{array}{c}0.25 \\
(-0.36,0.85)\end{array}$ & $\begin{array}{c}0.12 \\
(-0.25,0.48)\end{array}$ \\
\hline \multirow{3}{*}{$\begin{array}{l}\text { Age quartile } 2 \\
(31-38 \text { yo) } \\
N=422^{a}\end{array}$} & Q2 vs. Q1 & $\begin{array}{c}-0.65 \\
(-1.74,0.44)\end{array}$ & $\begin{array}{c}-1.09 \\
(-2.77,0.59)\end{array}$ & $\begin{array}{c}-0.38 \\
(-0.92,0.15)\end{array}$ & $\begin{array}{c}-0.21 \\
(-0.53,0.097)\end{array}$ \\
\hline & Q3 vs. Q1 & $\begin{array}{c}-1.34^{\star} \\
(-2.48,-0.19)\end{array}$ & $\begin{array}{c}-0.92 \\
(-2.63,0.78)\end{array}$ & $\begin{array}{c}-0.013 \\
(-0.68,0.41)\end{array}$ & $\begin{array}{c}-0.19 \\
(-0.51,0.13)\end{array}$ \\
\hline & Q4 vs. Q1 & $\begin{array}{c}-0.47 \\
(-1.69,0.75)\end{array}$ & $\begin{array}{c}-1.77^{\dagger} \\
(-3.67,0.13)\end{array}$ & $\begin{array}{c}-0.46 \\
(-1.06,0.13)\end{array}$ & $\begin{array}{c}-0.27 \\
(-0.62,0.086)\end{array}$ \\
\hline \multirow{3}{*}{$\begin{array}{l}\text { Age quartile } 3 \\
(39-51 \text { yo) } \\
N=506^{a}\end{array}$} & Q2 vs. Q1 & $\begin{array}{c}-0.43 \\
(-1.78,0.93)\end{array}$ & $\begin{array}{c}0.49 \\
(-1.25,2.24)\end{array}$ & $\begin{array}{c}0.024 \\
(-0.49,0.54)\end{array}$ & $\begin{array}{c}-0.039 \\
(-0.37,0.29)\end{array}$ \\
\hline & Q3 vs. Q1 & $\begin{array}{c}-0.83 \\
(-2.15,0.49)\end{array}$ & $\begin{array}{c}-0.21 \\
(-1.91,1.48)\end{array}$ & $\begin{array}{c}0.12 \\
(-0.38,0.62)\end{array}$ & $\begin{array}{c}-0.094 \\
(-0.42,0.23)\end{array}$ \\
\hline & Q4 vs. Q1 & $\begin{array}{c}-0.48 \\
(-1.79,0.83)\end{array}$ & $\begin{array}{c}0.78 \\
(-0.94,2.51)\end{array}$ & $\begin{array}{c}-0.067 \\
(-0.58,0.44)\end{array}$ & $\begin{array}{c}-0.023 \\
(-0.35,0.31)\end{array}$ \\
\hline \multirow{3}{*}{$\begin{array}{l}\text { Age quartile } 4 \\
(52-90 \text { yo) } \\
N=478^{a}\end{array}$} & Q2 vs. Q1 & $\begin{array}{c}0.20 \\
(-1.21,1.61)\end{array}$ & $\begin{array}{c}-0.025 \\
(-1.69,1.64)\end{array}$ & $\begin{array}{c}0.023 \\
(-0.47,0.51)\end{array}$ & $\begin{array}{c}0.019 \\
(-0.32,0.36)\end{array}$ \\
\hline & Q3 vs. Q1 & $\begin{array}{c}-0.31 \\
(-1.66,1.04)\end{array}$ & $\begin{array}{c}-0.69 \\
(-2.27,0.88)\end{array}$ & $\begin{array}{c}-0.12 \\
(-0.59,0.34)\end{array}$ & $\begin{array}{c}-0.15 \\
(-0.47,0.18)\end{array}$ \\
\hline & Q4 vs. Q1 & $\begin{array}{c}-0.62 \\
(-1.92,0.67)\end{array}$ & $\begin{array}{c}-1.29 \\
(-2.82,0.22)\end{array}$ & $\begin{array}{c}-0.45^{\star} \\
(-0.90,-0.004)\end{array}$ & $\begin{array}{c}-0.34^{\star} \\
(-0.65,-0.029)\end{array}$ \\
\hline
\end{tabular}

GAD-7, Generalized Anxiety Disorder Questionnaire 7-item; PHQ2, Patient Health Questionnaire- 2 item; Cl, confidence interval.

${ }^{a}$ The number of participants in each group is lower in multilevel model relative to Table $\mathbf{1}$ as only those participants who responded to all variables were included in the analysis.

${ }^{\dagger} P<0.1,{ }^{*} p<0.05$.

confounders that disproportionately affect younger adults during the pandemic like recent job loss and living alone, we did not control for participants having school-aged children. The nuances of these specific age groups could be studied further with larger populations and child-age could be included in future versions of the survey.

This study's main strength is the unique timing of the data collection. The surveyed population was living during a global pandemic with either lockdown precautions in place or social distancing recommendations put forth by global and national health organizations. These unusual conditions are likely to have a major impact on mental health (Brooks et al., 2020), and due to the social distancing precautions, the importance of greenspace is likely to be heightened. Additionally, we controlled for several demographic, mental health, and COVID-19 specific factors that allowed more precise assessment of the impact that greenspace provided. Finally, this study applied a robust assessment of treerich greenspaces based on the data collected and adequately accounted for tree canopy variation across the United States.

Several study limitations should be considered. These include a self-selection bias and a corresponding lack of diversity in our study population. The surveyed cohort consisted of an older, predominately white, female, wealthy, more highly educated, professional population that is enriched with healthcare providers and academics who decided to fill out a survey about mental health during this pandemic (Barzilay et al., 2020). COVID-19 has disproportionally impacted black communities and other communities of color enhancing our need to prioritize studies of greenspace and mental health in these populations (Yaya et al., 2020). This limitation is especially important as race was shown to moderate relationships between greenspace and mental health (Browning and Rigolon, 2018) and the racial disparity of COVID-19 infection was lower in areas with more greenspace ( $\mathrm{Lu}$ et al., 2021a), which could be explained by historical redlining leaving neighborhoods segregated and with unequal investments over time including access to greenspace (Nardone et al., 2021). Therefore, while we controlled for race as one of the covariates used in this analysis, more data is required from a more racially and socioeconomically diverse cohort to better understand the impact of greenspace on mental health during COVID-19 for these populations. Another key limitation is the inherent ascertainment bias in crowdsourcing data collection (Behrend et al., 2011), as this survey primarily reached academic populations in urban centers across the northeastern United States (Barzilay et al., 2020). These biases lessen the overall generalizability of this study and future work should be aimed at diversifying the population surveyed. 
With regard to our mental health outcomes, only brief screening measures of anxiety and depression were used in this study and more comprehensive measures could be considered in the future. With regard to the conceptualization of greenspace, we could only estimate available greenspace but could not account for the accessibility to, the quality of, or individual interactions with these places. Additionally, the spatial resolution of the analysis was limited to the ZIP Code by the identifiers gathered in the survey. Furthermore, the nature of using tree canopy data as a proxy for all greenspace in this study has several limitations including not accounting for other forms of urban greenery including grass, lawns and shrubbery that may be common in yards and residential settings important during this pandemic. The 30 $\times 30 \mathrm{~m}$ resolution of our tree canopy analysis also presents limitations in accounting for smaller clusters of trees that may be important for defining urban greenery. Another limitation is that we do not account for temporal changes in COVID19 spread, severity or variation in mitigation restrictions on social gatherings across ZIP Codes and along the course of data collection.

Additionally, the multiple comparisons made in the analyses should be interpreted with caution. Throughout the results we refer to effect estimates with $P$-values $<0.05$ as being statistically significant. It is important to note, however, that several additional effect estimates had $P$ values $<0.10$ and were considerable in magnitude. Here, we briefly draw attention to these results, which should be given some consideration to be consistent with the exploratory nature of this study given that greenspace has only minimally been investigated as potentially related to COVID-19-related mental health in the fashion done here. However, we present this data merely as exploratory to encourage future hypothesis driven research on the effects of greenspace on mental health during COVID-19. Lastly, the cross-sectional design and exploratory nature of this analysis limits the ability to draw causal inference, which can be addressed in future longitudinal studies with added measures of greenspace utilization.

We present data from a large opportunity sample collected during the first 6-months of the COVID-19 pandemic within the United States-a time when the majority of those sampled were restricted by social distancing precautions. We found a significant protective trend for greenspace on mental health, with exploratory analyses suggesting that mental health of certain age groups benefit more from proximity to greenspace than others. Longitudinal studies with more diverse cohorts and specific geographic identifiers are required to assess the causal relationship between greenspace and mental health during the COVID-19 pandemic. Nevertheless, it is apparent that now,

\section{REFERENCES}

Arroll, B., Goodyear-Smith, F., Crengle, S., Gunn, J., Kerse, N., Fishman, T., et al. (2010). Validation of PHQ-2 and PHQ-9 to screen for major depression in the primary care population. Ann. Fam. Med. 8, 348-353. doi: 10.1370/afm. 1139 more than ever, greenspace plays a unique role in preserving mental health.

\section{DATA AVAILABILITY STATEMENT}

The datasets presented in this article are not readily available because this study includes individual participants' data. Data cannot be publicly accessible due to Institutional Review Board guidelines. We are open to collaborations with other researchers upon contacting us. Requests to access the datasets should be directed to barzilayr@chop.edu.

\section{ETHICS STATEMENT}

The studies involving human participants were reviewed and approved by the Institutional Review Board of the University of Pennsylvania. The patients/participants provided their written informed consent to participate in this study.

\section{AUTHOR CONTRIBUTIONS}

Conceptualization: JW, RB, ES, VT, GD, EV, and DW. Methodology, investigation, resources, and writing-review and editing: JW, RB, ES, VT, GD, EV, DG, LB, RCG, REG, and DW. Software: JW, RB, ES, VT, GD, EV, DG, and DW. Validation: JW, DW, RB, and EV. Formal analysis: JW, DW, RB, ES, and EV. Data curation: RB, GD, EV, DG, LB, RCG, and REG. Writing-original draft preparation: JW. Visualization: JW, RB, and VT. Supervision: VT, DW, ES, and RB. Project administration and funding acquisition: RB. All authors have read and agreed to the published version of the manuscript.

\section{FUNDING}

This study was supported by the National Institute of Mental Health (NIMH) grants K23-MH120437 (RB), R01-MH119219 (REG, RCG), R01-MH117014 (RCG), and the Lifespan Brain Institute of Children's Hospital of Philadelphia and Penn Medicine, University of Pennsylvania. DG was funded in part by the Zuckerman STEM Leadership Program. The funding organizations had no role in the design and conduct of the study; collection, management, analysis, and interpretation of the data; preparation, review, or approval of the paper; and decision to submit the paper for publication.

\section{ACKNOWLEDGMENTS}

We thank participants of covid19resilience.org for their contribution to data generation.

Astell-Burt, T., and Feng, X. (2019). Association of urban green space with mental health and general health among adults in Australia. JAMA Netw. Open. 2:e198209. doi: 10.1001/jamanetworkopen.2019.8209

Astell-Burt, T., Mitchell, R., and Hartig, T. (2014). The association between green space and mental health varies across the lifecourse. A longitudinal study. J. Epidemiol. Commun. Health 68, 578-583. doi: 10.1136/jech-2013-203767 
Banerjee, D. (2020). The impact of Covid-19 pandemic on elderly mental health. Int. J. Geriatr. Psychiatry 35, 1466-1467. doi: 10.1002/gps.5320

Barzilay, R., Moore, T. M., Greenberg, D. M., DiDomenico, G. E., Brown, L. A., White, L. K., et al. (2020). Resilience, COVID-19-related stress, anxiety and depression during the pandemic in a large population enriched for healthcare providers. Transl. Psychiatry 10:291. doi: 10.1038/s41398-020-00982-4

Behrend, T. S., Sharek, D. J., Meade, A. W., and Wiebe, E. N. (2011). The viability of crowdsourcing for survey research. Behav. Res. Methods 43, 800-813. doi: 10.3758/s13428-011-0081-0

Bender, S., Goetz, W., Finco, M., Ruefenacht, B., Johnson, V., Reynolds, J., et al. (2019). 2016 Tree Canopy Cover for the National Land Cover Database : Production Data, Methods, Uses, and a Tour Through American Landscapes. 1, 5209910. USDA.

Beyer, K. M. M., Kaltenbach, A., Szabo, A., Bogar, S., Javier Nieto, F., and Malecki, K. M. (2014). Exposure to neighborhood green space and mental health: evidence from the survey of the health of Wisconsin. Int. J. Environ. Res. Public Health 11, 3453-3472. doi: 10.3390/ijerph110303453

Brooks, S. K., Webster, R. K., Smith, L. E., Woodland, L., Wessely, S., Greenberg, N., et al. (2020). The psychological impact of quarantine and how to reduce it: rapid review of the evidence. Lancet 395, 912-920. doi: 10.1016/S0140-6736(20)30460-8

Browning, M., and Lee, K. (2017). Within what distance does "greenness" best predict physical health? A systematic review of articles with gis buffer analyses across the lifespan. Int. J. Environ. Res. Public Health 14:675. doi: 10.3390/ijerph14070675

Browning, M. H. E. M., and Rigolon, A. (2018). Do income, race and ethnicity, and sprawl influence the greenspace-human health link in city-level analyses? Findings from 496 Cities in the United States. Int. J. Environ. Res. Public Health 15:1541. doi: 10.3390/ijerph15071541

Bryk, A. S., and Raudenbush, S. W. (1992). Advanced Qualitative Techniques in the Social Sciences, 1. Hierarchical Linear Models: Applications and Data Analysis Methods. Vol. 1. Thousand Oaks, CA: Sage Publication, Inc.

Cowie, H., and Myers, C. A. (2021). The impact of the COVID-19 pandemic on the mental health and well-being of children and young people. Child. Soc. 35, 62-74. doi: 10.1111/chso.12430

Cox, D. T. C., Shanahan, D. F., Hudson, H. L., Plummer, K. E., Siriwardena, G. M., Fuller, R. A., et al. (2017). Doses of neighborhood nature: the benefits for mental health of living with nature. BioScience 67, 147-155. doi: 10.1093/biosci/biw173

Engemann, K., Pedersen, C. B., Arge, L., Tsirogiannis, C., Mortensen, P. B., and Svenning, J. C. (2019). Residential green space in childhood is associated with lower risk of psychiatric disorders from adolescence into adulthood. Proc. Natl. Acad. Sci. U.S.A. 116, 5188-5193. doi: 10.1073/pnas.1807504116

ESRI (2011). ArcGIS Desktop: Release 10. Environmental Systems Research Institute.

Francis, J., Wood, L. J., Knuiman, M., and Giles-Corti, B. (2012). Quality or quantity? Exploring the relationship between Public Open Space attributes and mental health in Perth, Western Australia. Soc. Sci. Med. 74, 1570-1577. doi: $10.1016 /$ j.socscimed.2012.01.032

Gao, G., and Sai, L. (2020). Towards a 'virtual' world: social isolation and struggles during the COVID-19 pandemic as single women living alone. Gender Work Organ. 27, 754-762. doi: 10.1111/gwao. 12468

Gobbi, S., Płomecka, M. B., Ashraf, Z., Radziński, P., Neckels, R., Lazzeri, S., et al. (2020). Worsening of preexisting psychiatric conditions during the COVID-19 pandemic. Front. Psychiatry 11:581426. doi: 10.3389/fpsyt.2020.581426

Hedblom, M., Gunnarsson, B., Iravani, B., Knez, I., Schaefer, M., Thorsson, P., et al. (2019). Reduction of physiological stress by urban green space in a multisensory virtual experiment. Sci. Rep. 9, 1-11. doi: 10.1038/s41598-019-46099-7

Hiraoka, D., and Tomoda, A. (2020). Relationship between parenting stress and school closures due to the COVID-19 pandemic. Psychiatry Clin. Neurosci. 74, 497-498. doi: 10.1111/pcn.13088

Joliffe, I. (1992). Principal component analysis and exploratory factor analysis. Stat. Methods Med. Res. 1, 69-95. doi: 10.1177/096228029200100105

Killgore, W. D. S., Cloonan, S. A., Taylor, E. C., and Dailey, N. S. (2020). Loneliness: a signature mental health concern in the era of COVID-19. Psychiatry Res. 290:113117. doi: 10.1016/j.psychres.2020.113117

Lu, Y., Chen, L., Liu, X., Yang, Y., Sullivan, W. C., Xu, W., et al. (2021a). Green spaces mitigate racial disparity of health: a higher ratio of green spaces indicates a lower racial disparity in SARS-CoV-2 infection rates in the USA. Environ. Int. 152:106465. doi: 10.1016/j.envint.2021.106465
Lu, Y., Zhao, J., Wu, X., and Lo, S. M. (2021b). Escaping to nature during a pandemic: a natural experiment in Asian cities during the COVID19 pandemic with big social media data. Sci. Total Environ. 777:146092. doi: 10.1016/j.scitotenv.2021.146092

Marchini, S., Zaurino, E., Bouziotis, J., Brondino, N., Delvenne, V., and Delhaye, M. (2020). Study of resilience and loneliness in youth (18-25 years old) during the COVID-19 pandemic lockdown measures. J. Community Psychol. 49, 468-480. doi: $10.1002 /$ jcop. 22473

Mazumdar, S., Dunshea, A., Chong, S., and Jalaludin, B. (2020). Tree canopy cover is best associated with perceptions of greenspace: a short communication. Int. J. Environ. Res. Public Health 17, 1-9. doi: 10.3390/ijerph17186501

Moreland, A., Herlihy, C., Tynan, M. A., Sunshine, G., McCord, R. F., Hilton, C., et al. (2020). Timing of state and territorial COVID-19 stay-at-home orders and changes in population movement - United States, March 1-May 31, 2020. Morbid. Mortal. Week. Rep. 69, 1198-1203. doi: 10.15585/mmwr.mm6935a2

Naomi, A. S. (2020). Access to nature has always been important; with COVID-19, it is essential. Health Environ. Res. Des. J. 13, 242-244. doi: $10.1177 / 1937586720949792$

Nardone, A., Rudolph, K. E., Morello-Frosch, R., and Casey, J. A. (2021). Redlines and greenspace: the relationship between historical redlining and 2010 greenspace across the United States. Environ. Health Perspect. 129, 1-9. doi: $10.1289 /$ EHP7495

Nutsford, D., Pearson, A. L., and Kingham, S. (2013). An ecological study investigating the association between access to urban green space and mental health. Public Health 127, 1005-1011. doi: 10.1016/j.puhe.2013.08.016

Panchal, N., Kamal, R., Orgera, K., Cox, C., Garfield, R., Hamel, L., et al. (2020). The Implications of COVID-19 for Mental Health and Substance Use. KFF. Kaiser Family Foundation, 1-11.

Pouso, S., Borja, Á., Fleming, L. E., Gómez-Baggethun, E., White, M. P., and Uyarra, M. C. (2020). Contact with blue-green spaces during the COVID19 pandemic lockdown beneficial for mental health. Sci. Total Environ. 756:143984. doi: 10.1016/j.scitotenv, 0.2020 .143984

R Studio Team (2020). RStudio: Integrated Development. Boston, MA: RStudio, PBC.

Ravens-Sieberer, U., Kaman, A., Erhart, M., Devine, J., Schlack, R., and Otto, C. (2021). Impact of the COVID-19 pandemic on quality of life and mental health in children and adolescents in Germany. Eur. Child Adolesc. Psychiatry. 1-11. doi: 10.1007/s00787-021-01726-5

Rossi, R., Socci, V., Talevi, D., Mensi, S., Niolu, C., Pacitti, F., et al. (2020). COVID-19 pandemic and lockdown measures impact on mental health among the general population in italy. Front. Psychiatry 11:790. doi: $10.3389 /$ fpsyt. 2020.00790

Ruijsbroek, A., Mohnen, S. M., Droomers, M., Kruize, H., Gidlow, C. GraŽulevičiene, R., et al. (2017). Neighbourhood green space, social environment and mental health: an examination in four European cities. Int. J. Public Health 62, 657-667. doi: 10.1007/s00038-017-0963-8

Rural-Urban Continuum Codes (2013). Economic Research Service United States Department of Agriculture. Available online at: https://www.ers.usda.gov/dataproducts/rural-urban-continuum-codes.aspx (accessed January 12, 2021).

Slater, S. J., Christiana, R. W., and Gustat, J. (2020). Recommendations for keeping parks and green space accessible for mental and physical health during COVID-19 and other pandemics. Prevent. Chronic Dis. 17:E59. doi: 10.5888/PCD17.200204

Soga, M., Evans, M. J., Tsuchiya, K., and Fukano, Y. (2020). A room with a green view: the importance of nearby nature for mental health during the COVID-19 pandemic. Ecol. Appl. 31:e2248. doi: 10.1002/eap. 2248

South, E. C., Hohl, B. C., Kondo, M. C., MacDonald, J. M., and Branas, C. C. (2018). Effect of greening vacant land on mental health of communitydwelling adults: a cluster randomized trial. JAMA Network Open 1:e180298. doi: 10.1001/jamanetworkopen.2018.0298

Spitzer, R. L., Kroenke, K., Williams, J. B. W., and Löwe, B. (2006). A brief measure for assessing generalized anxiety disorder: the GAD-7. Arch. Intern. Med. 166, 1092-1097. doi: 10.1001/archinte.166.10.1092

StataCorp. (2019). Stata Statistical Software: Release 16. StataCorp LLC.

Twohig-Bennett, C., and Jones, A. (2018). The health benefits of the great outdoors: a systematic review and meta-analysis of greenspace exposure and health outcomes. Environ. Res. 166, 628-637. doi: 10.1016/j.envres.2018. 06.030 
Ueda, M., Stickley, A., Sueki, H., and Matsubayashi, T. (2020). Mental health status of the general population in japan during the COVID-19 pandemic. Psychiatry Clin. Neurosci. 74, 505-506. doi: 10.1111/pcn.13105

Venter, Z. S., Barton, D. N., Gundersen, V., Figari, H., and Nowell, M. (2020). Urban nature in a time of crisis: recreational use of green space increases during the COVID-19 outbreak in Oslo, Norway. Environ. Res. Lett. 15:104075. doi: $10.1088 / 1748-9326 /$ abb396

Vindegaard, N., and Benros, M. E. (2020). COVID-19 pandemic and mental health consequences: Systematic review of the current evidence. Brain Behav. Immun. 89, 531-542. doi: 10.1016/j.bbi.2020.05.048

Witteveen, D., and Velthorst, E. (2020). Economic hardship and mental health complaints during COVID-19. Proc. Natl. Acad. Sci. U.S.A. 117, 27277-27284. doi: 10.1073/pnas.2009609117

Yaya, S., Yeboah, H., Charles, C. H., Otu, A., and Labonte, R. (2020). Ethnic and racial disparities in COVID-19-related deaths: counting the trees, hiding the forest. BMJ Global Health 5, 1-5. doi: 10.1136/bmjgh-2020-002913

Ziersch, A. M., Baum, F., Woodman, R. J., Newman, L., and Jolley, G. (2014). A longitudinal study of the mental health impacts of job loss: the role of socioeconomic, sociodemographic, and social capital factors. J. Occup. Environ. Med. 56, 714-720. doi: 10.1097/JOM.0000000000 000193
Conflict of Interest: RB serves on the scientific board and reports stock ownership in "Taliaz Health", with no conflict of interest relevant to this work.

The remaining authors declare that the research was conducted in the absence of any commercial or financial relationships that could be construed as a potential conflict of interest.

Publisher's Note: All claims expressed in this article are solely those of the authors and do not necessarily represent those of their affiliated organizations, or those of the publisher, the editors and the reviewers. Any product that may be evaluated in this article, or claim that may be made by its manufacturer, is not guaranteed or endorsed by the publisher.

Copyright (๑) 2021 Wortzel, Wiebe, DiDomenico, Visoki, South, Tam, Greenberg, Brown, Gur, Gur and Barzilay. This is an open-access article distributed under the terms of the Creative Commons Attribution License (CC BY). The use, distribution or reproduction in other forums is permitted, provided the original author $(s)$ and the copyright owner(s) are credited and that the original publication in this journal is cited, in accordance with accepted academic practice. No use, distribution or reproduction is permitted which does not comply with these terms. 


\section{APPENDIX}

TABLE A1 | Preliminary Greenspace buffer analysis to determine optimal buffer size.

\begin{tabular}{|c|c|c|c|c|c|}
\hline & $\begin{array}{c}\text { No buffer } \\
\text { Coef. }(95 \% \text { Cl) }\end{array}$ & $\begin{array}{l}100 \mathrm{~m} \text { buffer } \\
\text { Coef. }(95 \% \mathrm{Cl})\end{array}$ & $\begin{array}{l}250 \mathrm{~m} \text { buffer } \\
\text { Coef. (95\% Cl) }\end{array}$ & $\begin{array}{l}500 \mathrm{~m} \text { buffer } \\
\text { Coef. (95\% Cl) }\end{array}$ & $\begin{array}{l}1,000 \mathrm{~m} \text { buffer } \\
\text { Coef. }(95 \% \mathrm{Cl})\end{array}$ \\
\hline COVID-19 worries & $\begin{array}{c}-0.0098 \\
(-0.023,0.005)\end{array}$ & $\begin{array}{c}-0.011 \\
(-0.025,0.003)\end{array}$ & $\begin{array}{c}-0.011 \\
(-0.03,0.003)\end{array}$ & $\begin{array}{c}-0.012 \\
(-0.026,0.002)\end{array}$ & $\begin{array}{c}-0.009 \\
(-0.024,0.005)\end{array}$ \\
\hline GAD-7 & $\begin{array}{c}-0.005 \\
(-0.03,0.017)\end{array}$ & $\begin{array}{c}-0.008 \\
(-0.029,0.013)\end{array}$ & $\begin{array}{c}-0.009 \\
(-0.03,0.013)\end{array}$ & $\begin{array}{c}-0.009 \\
(-0.03,0.012)\end{array}$ & $\begin{array}{c}-0.008 \\
(-0.03,0.013)\end{array}$ \\
\hline PHQ-2 & $\begin{array}{c}-0.0015 \\
(-0.008,0.005)\end{array}$ & $\begin{array}{c}-0.003 \\
(-0.009,0.004)\end{array}$ & $\begin{array}{c}-0.003 \\
(-0.009,0.003)\end{array}$ & $\begin{array}{c}-0.003 \\
(-0.009,0.004)\end{array}$ & $\begin{array}{c}-0.002 \\
(-0.009,0.004)\end{array}$ \\
\hline
\end{tabular}

GAD-7, Generalized Anxiety Disorder Questionnaire 7-item; PHQ2, Patient Health Questionnaire- 2 item; Cl, confidence interval. Greenspace was defined as a continuous variable. Subsequent analysis stratified greenspace by quartiles.

${ }^{\dagger} P<0.1$.

TABLE A2 | Full multivariate regression analysis with multilevel models of all covariates.

\begin{tabular}{|c|c|c|c|c|c|}
\hline Covariates & $\begin{array}{l}\text { Categorical } \\
\text { breakdown }\end{array}$ & $\begin{array}{c}\text { COVID-19 related } \\
\text { worries } \\
\text { Coef. }(95 \% \mathrm{Cl})\end{array}$ & $\begin{array}{c}\text { GAD-7 } \\
\text { Coef. }(95 \% \mathrm{CI})\end{array}$ & $\begin{array}{c}\text { PHQ-2 } \\
\text { Coef. }(95 \% \text { Cl) }\end{array}$ & $\begin{array}{l}\text { Composite mental } \\
\text { health score } \\
\text { Coef. }(95 \% \mathrm{Cl})\end{array}$ \\
\hline Sex & & $-1.11^{\star \star}(-1.66,-0.57)$ & $-0.76^{\dagger}(-1.56,0.04)$ & $0.07(-0.17,0.31)$ & $-0.12(-0.19,0.20)$ \\
\hline \multirow[t]{2}{*}{ Age } & 1 & $0.41(-0.27,1.09)$ & $-0.13(-1.14,0.87)$ & $-0.11(-0.42,0.19)$ & $0.001(-0.19,0.21)$ \\
\hline & 4 & $0.11(-0.55,0.77)$ & $-1.93^{*}(-2.90,-0.96)$ & $-0.25^{\dagger}(-0.54,0.04)$ & $-0.22^{*}(-0.40,-0.03)$ \\
\hline \multirow[t]{4}{*}{ Edu } & 2 & $8.89^{\star}(2.65,15.12)$ & $-9.54^{\dagger}(-19.2,0.11)$ & $-2.64^{\dagger}(-5.56,0.29)$ & $-0.51(-2.38,1.36)$ \\
\hline & 3 & $7.39^{\star}(1.26,13.5)$ & $-11.1^{*}(-20.6,-1.58)$ & $-3.66^{\star}(-6.53,-0.78)$ & $-1.05(-2.89,0.79)$ \\
\hline & 4 & $7.36^{\star}(11.25,13.4)$ & $-11.8^{*}(-20.9,-2.02)$ & $-4.04^{\star}(-6.91,-1.18)$ & $-1.22(-3.05,0.61)$ \\
\hline & 5 & $6.55^{\star}(0.44,12.7)$ & $-11.8^{*}(-21.2,-2.35)$ & $-4.25^{\star}(-7.11,-1.38)$ & $-1.35(-3.19,0.48)$ \\
\hline Recent job loss & & $1.56^{*}(0.51,0.2 .60)$ & $-0.43(-2.03,1.17)$ & $0.81^{\star}(0.33,1.29)$ & $0.31(-0.002,0.62)$ \\
\hline Lives alone & & $-0.38(-0.89,0.13)$ & $-0.09(-0.83,0.64)$ & $0.29^{\star}(0.07,0.52)$ & $0.03(-0.11,0.18)$ \\
\hline \multirow[t]{7}{*}{ Urbanicity } & 2 & $-0.15(-0.78,0.48)$ & $0.30(-0.59,1.19)$ & $0.11(-0.16,0.38)$ & $0.04(-0.14,0.21)$ \\
\hline & 3 & $-1.59^{\star}(-2.90,-0.28)$ & $-0.68(-2.55,1.20)$ & $0.11(-0.45,0.68)$ & $-0.17(-0.53,0.19)$ \\
\hline & 4 & $-0.05(-2.89,-0.28)$ & $-0.02(-3.41,3.37)$ & $0.45(-0.58,1.47)$ & $0.12(-0.53,0.78)$ \\
\hline & 5 & $-0.43(-1.89,6.76)$ & $6.63^{*}(1.12,12.12)$ & $2.10^{\star}(0.45,3.76)$ & $1.48^{\star}(0.51,1.15)$ \\
\hline & 6 & $1.45(-1.62,4.51)$ & $2.52(-1.78,6.83)$ & $0.09(-1.21,1.38)$ & $0.32(-0.51,1.15)$ \\
\hline & 7 & $-2.73^{\star}(-5.25,-0.21)$ & $-0.98(-4.38,2.42)$ & $-0.03(-1.05,0.99)$ & $-0.22(-0.87,0.44)$ \\
\hline & 8 & $2.47(-1.39,6.34)$ & $3.14(-2.37,8.65)$ & $0.95(-0.71,2.61)$ & $0.65(-0.42,1.71)$ \\
\hline
\end{tabular}

GAD-7, Generalized Anxiety Disorder Questionnaire 7-item; PHQ2, Patient Health Questionnaire- 2 item; Cl, confidence interval. Greenspace was defined as a continuous variable. Subsequent analysis stratified greenspace by quartiles.

${ }^{a}$ The number of participants in each group is lower in multilevel model relative to Table $\mathbf{1}$ as only those participants who responded to all variables were included in the analysis.

${ }^{\dagger} P<0.1,{ }^{*} p<0.05,{ }^{* *} p<0.0001$ 